\title{
Structure Analysis and Optimization for Compacting System of Asphalt Paver
}

\author{
Sun Jian*
}

\author{
Mechanical and Electronic Department, Xuzhou Institute of Technology, Jiangsu Key Laboratory of Large Engineering \\ Equipment Detection and Control, Xuzhou 221111, China
}

\begin{abstract}
Compound motion of double-tamper mechanism causes an impact force on the frame of flatiron-box, which affects the quality of the load when the asphalt paver is running. Different from the previous simplified model, in this paper, the double-tamper mechanism with two parallel slider-cranks is considered. According to the analysis of the structure and principle of the mechanism, and considering the size and mass distribution of each component, the inertia force balance optimization for the double vibrating mechanism is calculated. According to the results of optimization, the vibration experiments were carried out on real screed. The experimental results show that, the harmful inertial force produced by the dual tamping mechanism has been significantly reduced, and the stability of vibration can be effectively improved when screed is paving. The research could provide reliable theoretical method and basis for design, manufacturing and use of asphalt concrete paver.
\end{abstract}

Keywords: Asphalt paver, Optimization, Structure analysis, Tamper mechanism, Concrete paver, Slider-crank mechanism.

\section{INTRODUCTION}

Compacting system is the key device for asphalt concrete paver. It achieves modeling, leveling and compaction of paving roads [1]. The screed with double-tamper mechanism has been widely applied in the market due to the characteristics of good leveling high density and high operating efficiency. The double-tamper mechanism in the front of the screed is driven by the rotation of the vibrating beams, and its rotational frequency is commonly between 0 $\sim 25 \mathrm{~Hz}$. The right choice of the structure and dynamics parameters of compacting system related to the paver cause it to reliably. The tamper and the eccentric vibration mechanism of screed directly affect the paving flatness and compactness. Experimental studies have found that the tampering will produce harmful impact to screed, which leads to uneven paving of roads and uneven pre-compaction. In some early researches, the movement of the tamper mechanism is simplified as simple harmonic vibration and didn't take the vibration of the vibrating beams into account [2]. Few studies [3] have investigated the theoretical calculation based on the method of complex vector. The process of this method is trivial and only the movement parameters of the tamper mechanism can be calculated.

In this paper, the structure and working principle of tamper mechanism are analyzed, and then the double-tamper with two parallel slider-cranks mechanism is analyzed, considering the size and mass distribution of each component. The inertia force balance analysis was carried out on the double tamper mechanism, optimizing its structure parameters, and reducing its impact on the screed vibration.

*Address correspondence to this author at the Mechanical and Electronic Department, Xuzhou Institute of Technology, Jiangsu Key Laboratory of Large Engineering Equipment Detection and Control, Xuzhou 221111, China; Tel: +86-15895232391; E-mail: sunjian751@163.com

$1874-155 X / 15$

\section{STRUCTURE AND WORK PRINCIPLE OF THE TAMPER MECHANISM}

The structure of double-tamper mechanism of screed is shown in Fig. (1), and the main vibrating parts include: the eccentric shaft, main vibrating beam, auxiliary vibrating beam, main eccentric sleeve and auxiliary eccentric sleeve [4]. The initial compaction of the paving mixture is realized by up and down movement of the vibration beam which is caused by the rotation of the eccentric shaft. The main and auxiliary vibrating beams are considered as the slab and girder structure. The top of the beams are rigid, and the beams are hanged on the eccentric shaft.

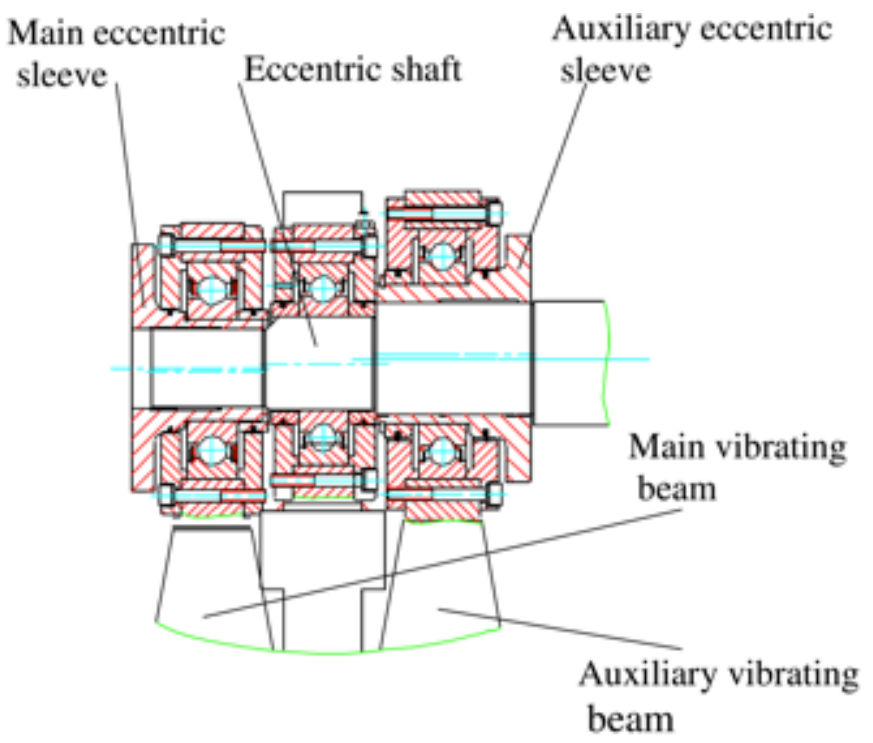

Fig. (1). Structure type of double-tamper mechanism.

The power of tamper mechanism is supplied by hydraulic motor, speed can be adjusted by a rotary flow speed control 
valve and the working speed ranges from $0 \sim 1500 \mathrm{r} / \mathrm{min}$. Amplitude is varied by adjusting rotation angle of the main and auxiliary eccentric sleeves [5]. The eccentric shaft is a power transmission part of tamper mechanism and the main mechanism of amplitude generation, which is shown in Fig. (2).

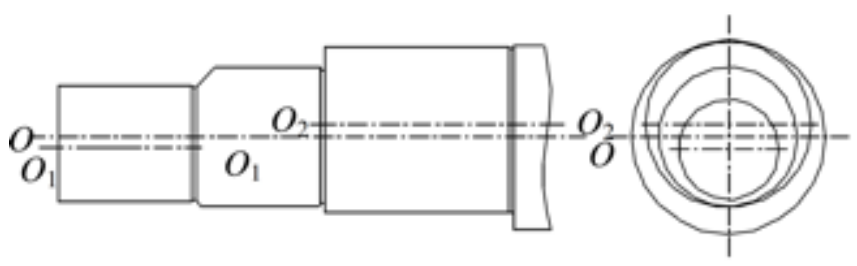

Fig. (2). Structure of eccentric shaft.

In Fig. (2), $O-O$ is the rotary center line of eccentric shaft, $O_{1}-O_{1}$ is the eccentric center line of main vibration, and $\mathrm{O}_{2}-\mathrm{O}_{2}$ is the eccentric center of auxiliary vibration. When the eccentric shaft rotates about the rotation center line $O-O$, the $O_{1}-O_{1}$ and $O_{2}-O_{2}$ eccentric center lines do the eccentric movement around $O-O$ respectively. The eccentric motion is the movement source of composite motion for the eccentric shaft and eccentric sleeve. The main and auxiliary eccentric sleeves are also eccentric parts. The main eccentric sleeve is installed on the eccentric shaft $O_{1}-O_{1}$ and is used for the amplitude adjustment of the vibrator's main vibrating beam. The auxiliary eccentric sleeve is installed on the eccentric shaft $\mathrm{O}_{2}-\mathrm{O}_{2}$ and used for amplitude adjustment of vibrator's auxiliary vibrating beam. The structure of main and auxiliary eccentric sleeve are similar, and the schematic diagram of the eccentric sleeve is shown in Fig. (3).
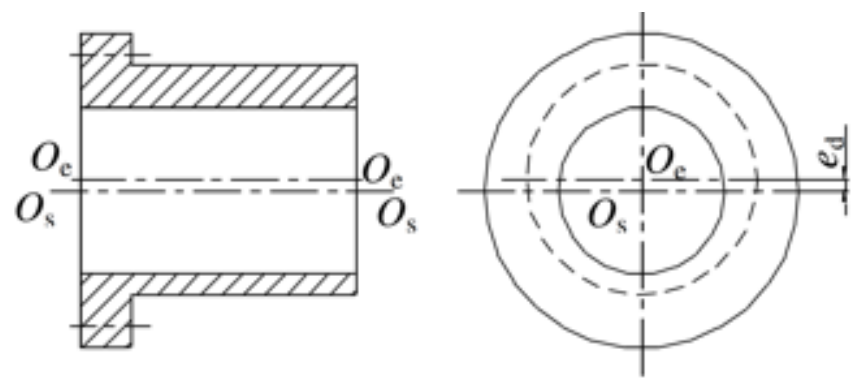

Fig. (3). Structure of eccentric sleeve.

In Fig. (3), $O_{\mathrm{s}}-O_{\mathrm{s}}$ is the rotary center line of eccentric sleeve, $O_{\mathrm{e}}-O_{\mathrm{e}}$ is the eccentric center line of eccentric sleeve, and $e_{\mathrm{d}}$ is the eccentric quantity. Amplitude change is realized through adjusting rotation angle of the sleeve that is arranged on the eccentric shaft. There are some markers in the surrounding of the eccentric sleeve. When rotating the eccentric sleeve to make the markers align with the scribed line on the connecting flange, which can obtain the required amplitude. The variety of different combinations of amplitudes can be obtained by adjusting the eccentricity of the eccentric shaft and eccentric sleeve, and also different phase differences can be achieved between the the main and auxiliary vibrating beams.

The amplitude of double-tamper mechanism has a variety of combinations [6]. A certain company's screed vibrator mechanism is used for analysis in the following example. The main vibration amplitude of the screed is 3, 5, 7 and 9 $\mathrm{mm}$, and the auxiliary vibrating amplitude is $0,3,6,9$ and 12 $\mathrm{mm}$. The adjustment method of the amplitude has many kinds of combinations. According to the structure principle of tamper mechanism, different amplitude combinations of main and auxiliary vibrating beam are shown in Fig. (4).

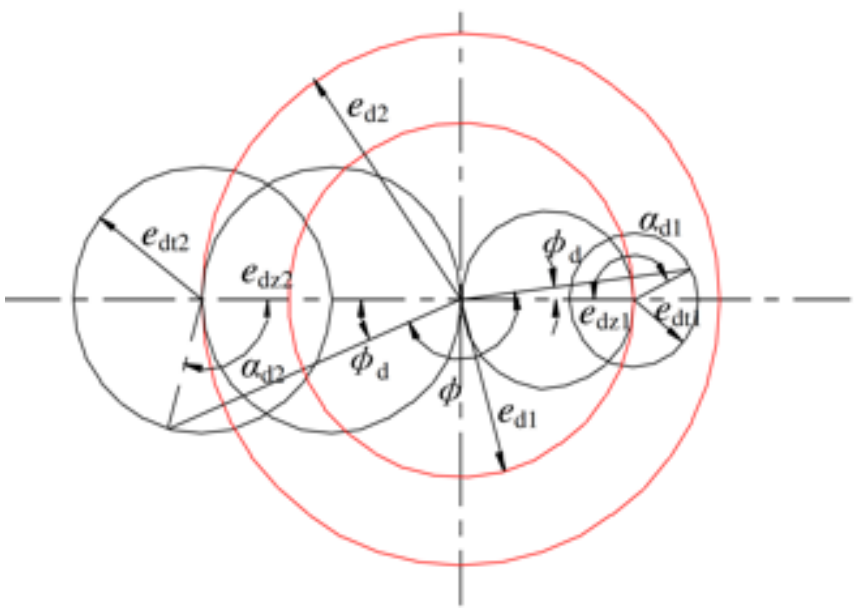

Fig. (4). Amplitude combination of the double tampers.

In Fig. (4), $e_{\mathrm{d} 1}$ is the main vibrator eccentricity, $e_{\mathrm{d} 2}$ is the auxiliary vibrator eccentricity, $e_{\mathrm{dz} 1}$ is the the eccentric quantity of the main vibrating shaft neck, $e_{\mathrm{dt}}$ is the eccentric quantity of main eccentric sleeve, $e_{\mathrm{dz} 2}$ is the eccentric quantity of the auxiliary vibrating shaft neck, and $e_{\mathrm{d} 2}$ is the eccentric quantity of auxiliary eccentric sleeve.

According to Fig. (4), the eccentricity of main vibrating mechanism can be expressed as follows:

$e_{d 1}=\sqrt{e_{d z 1}^{2}+e_{d t 1}^{2}-2 e_{d z 1} e_{d t 1} \cos \alpha_{d 1}}$

The eccentricity of auxiliary vibrating mechanism is

$e_{d 2}=\sqrt{e_{d z 2}{ }^{2}+e_{d t 2}{ }^{2}-2 e_{d z 2} e_{d t 2} \cos \alpha_{d 2}}$

According to the trigonometric relationships shown in Fig. (3), the geometric equations can be obtained as follows:

$$
\left.\begin{array}{l}
\cos \phi_{d 1}=\frac{e_{d 1}{ }^{2}+e_{d z 1}{ }^{2}-e_{d t 1}{ }^{2}}{2 e_{d 1} e_{d z 1}} \\
\cos \phi_{d 2}=\frac{e_{d 2}{ }^{2}+e_{d z 2}{ }^{2}-e_{d t 2}{ }^{2}}{2 e_{d 2} e_{d z 2}}
\end{array}\right\}
$$

The relationship between amplitude and the corresponding phase angle of main and auxiliary vibrating beam is as follows:

$$
\begin{aligned}
\phi= & 180^{\circ}+\phi_{d 1}-\phi_{d 2} \\
= & 180^{\circ}+\cos ^{-1}\left(\frac{e_{d 1}{ }^{2}+e_{d z 1}{ }^{2}-e_{d t 1}{ }^{2}}{2 e_{d 1} e_{d z 1}}\right) \\
& -\cos ^{-1}\left(\frac{e_{d 2}{ }^{2}+e_{d z 2}{ }^{2}-e_{d t 2}{ }^{2}}{2 e_{d 2} e_{d z 2}}\right)
\end{aligned}
$$

By equation 4, we can get that phase Angle between the main and the auxiliary vibrating beam is determined by the amplitude of the main and the auxiliary vibrating beam. Given a set of amplitude values, we will determine a fixed Phase Angle value. According to the main and auxiliary vibrating stroke adjusted working stroke, there are 20 kinds 
Table 1. Phase between the main and auxiliary tamper.

\begin{tabular}{|c|c|c|c|c|c|}
\hline SN & Stroke/mm & Phase Angle/( $\left(^{\circ}\right)$ & SN & Stroke/mm & Phase Angle $/\left({ }^{\circ}\right)$ \\
\hline 1 & 3,0 & 180 & 11 & 7,0 & 153.4 \\
\hline 2 & 3,3 & 255 & 12 & 7,3 & 228.4 \\
\hline 3 & 3,6 & 240 & 13 & 7,6 & 213.4 \\
\hline 4 & 3,9 & 225 & 14 & 7,9 & 198.4 \\
\hline 5 & 3,12 & 180 & 15 & 7,12 & 153.4 \\
\hline 6 & 5,0 & 150 & 16 & 9,0 & 180 \\
\hline 7 & 5,3 & 225 & 17 & 9,3 & 255 \\
\hline 8 & 5,6 & 210 & 18 & 9,6 & 240 \\
\hline 9 & 5,9 & 195 & 19 & 9,9 & 225 \\
\hline 10 & 5,12 & 150 & 20 & 9,12 & 180 \\
\hline
\end{tabular}

of state. The calculated phase angle corresponding to each state between main and auxiliary vibrating beam is shown in Table 1.

\section{STRUCTURE OPTIMIZATION}

Kinematics essence of tamper mechanism can be identified as two sets of offset slider-crank mechanism in parallel. When operating, the eccentric shaft rotation produces a large inertial impact force which will cause the vibration of the screed [7]. The main role of the double tamper mechanism is preliminary ramming material, so its influence on the screed would be eliminated. The main and auxiliary vibrating beam's task is to partially offset the inertial force. However, the current design of the tamper mechanism is only a simple imitation of imported equipment and there is no analysis of its calculation. So the design of the tamper beam sometimes increases the inertia force of mechanism, which will have great influence on the paving quality of the screed. For mechanical components with reciprocating motion or general plane motion, the balance problem is generally more complex, often only part of the balance of inertia force is obtained. In this paper, the method of planar mechanism balance is adopted to reduce the vibration of the double vibrating mechanism [8].

The calculation model of the structure of the double tamper mechanism is shown in Fig. (5).

Where $G_{\mathrm{d} 1} \sim G_{\mathrm{d} 4}$ indicate each centroid of the four rigid body that are main and auxiliary vibrating beam, main and auxiliary eccentric sleeve, $\theta_{\mathrm{d} 1}$ is the initial phase angle of the main vibration, $\theta_{\mathrm{d} 2}$ is the initial phase angle of the auxiliary vibrator, $\theta_{\mathrm{d} 3}$ is the angle between the centroid symmetry plane and the vertical plane of main vibrating beam, $\theta_{\mathrm{d} 4}$ is the angle between the centroid symmetry plane and the vertical plane of auxiliary vibrating beam, $\Phi$ is the phase difference between main vibrating beam and auxiliary vibrating beam, $a_{1}$ is the distance from the centroid to eccentric center of main vibrating beam, $a_{2}$ is the distance from the centroid to eccentric center of auxiliary vibrating beam, $a_{3}$ is the distance from symmetry plane of centroid center to eccentric center of main vibrating beam, $a_{4}$ is the distance from symmetry plane of centroid center to eccentric center of

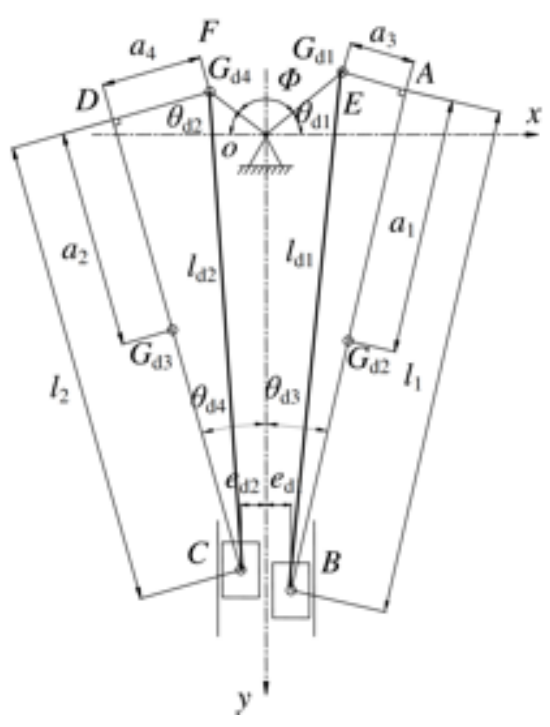

Fig. (5). Calculation model of double-tamper mechanism.

auxiliary vibrating beam, $e_{\mathrm{d} 1}$ is the distance from symmetry plane of centroid center to rotation center of main vibrating beam, $e_{\mathrm{d} 2}$ is the distance from symmetry plane of centroid center to rotation center of auxiliary vibrating beam, $l_{1}$ is the height of the main vibrating beam, $l_{2}$ is the height of the auxiliary vibrating beam.

Assume that the eccentric shaft is in uniform motion around the axis $O$ with angular velocity $\omega_{\mathrm{d}} . O E$ is the crank radius of main vibration, $|O E|=r_{\mathrm{d} 1}, E B$ is the connecting rod, $|E B|=l_{\mathrm{d} 1}=\sqrt{l_{1}^{2}+a_{3}^{2}}, \varepsilon_{\mathrm{d} 1}$ is the main vibrator eccentricity, $\varepsilon_{\mathrm{d} 1}=e_{\mathrm{d} 1} / r_{\mathrm{d} 1}$. Then the end displacement of main vibration beam can be expressed as follows:

$$
\begin{aligned}
y_{\mathrm{d} 1}=l_{\mathrm{d} 1} & -r_{\mathrm{d} 1} \cos \omega_{\mathrm{d}} t-\lambda_{\mathrm{d} 1} e_{\mathrm{d} 1} \sin \omega_{\mathrm{d}} t \\
& +\frac{\lambda_{\mathrm{d} 1} r}{4}\left(1-\cos 2 \omega_{\mathrm{d}} t\right)+\frac{1}{2\left(1+\lambda_{\mathrm{d} 1}\right)} \lambda_{\mathrm{d} 1}^{2} e_{\mathrm{d} 1}^{2}
\end{aligned}
$$

where $\lambda_{\mathrm{d} 1}$ is the crank connecting rod ratio of main vibration, $\lambda_{\mathrm{d} 1}=r_{\mathrm{d} 1} / l_{\mathrm{d} 1}$. 
The main vibrating beam end speed can be expressed as:

$$
\dot{y}_{\mathrm{d} 1}=-r_{\mathrm{d} 1} \omega_{\mathrm{d}}\left(\sin \omega_{\mathrm{d}} t+\lambda_{\mathrm{d} 1} \varepsilon_{\mathrm{d} 1} \cos \omega_{\mathrm{d}} t+\frac{\lambda_{\mathrm{d} 1}}{2} \sin 2 \omega_{\mathrm{d}} t\right)
$$

The main vibrating beam end acceleration can be expressed as:

$$
\ddot{y}_{\mathrm{d} 1}=-r_{\mathrm{d} 1} \omega_{\mathrm{d}}^{2}\left(\cos \omega_{\mathrm{d}} t-\lambda_{\mathrm{d} 1} \varepsilon_{\mathrm{d} 1} \sin \omega_{\mathrm{d}} t+\lambda_{\mathrm{d} 1} \cos 2 \omega_{\mathrm{d}} t\right)
$$

$O F$ is the crank radius of auxiliary vibration, $|O F|=r_{\mathrm{d} 2}$, $F C$ is the connecting $\operatorname{rod},|F C|=l_{\mathrm{d} 2}=\sqrt{l_{2}^{2}+a_{4}^{2}}, \varepsilon_{\mathrm{d} 2}$ is the main vibrator eccentricity, $\varepsilon_{\mathrm{d} 2}=e_{\mathrm{d} 2} / r_{\mathrm{d} 2}$. Then the end displacement of auxiliary vibration beam can be expressed as follows:

$$
\begin{aligned}
y_{\mathrm{d} 2}= & l_{\mathrm{d} 2}-r_{\mathrm{d} 2} \cos \omega_{\mathrm{d}} t-\lambda_{\mathrm{d} 2} e_{\mathrm{d} 2} \sin \omega_{\mathrm{d}} t \\
& +\frac{\lambda_{\mathrm{d} 2} r}{4}\left(1-\cos 2 \omega_{\mathrm{d}} t\right)+\frac{1}{2\left(1+\lambda_{\mathrm{d} 2}\right)} \lambda_{\mathrm{d} 2}^{2} e_{\mathrm{d} 2}^{2}
\end{aligned}
$$

The auxiliary vibrating beam end speed is expressed as:

$\dot{y}_{\mathrm{d} 2}=-r_{\mathrm{d} 2} \omega_{\mathrm{d}}\left(\sin \omega_{\mathrm{d}} t+\lambda_{\mathrm{d} 2} \varepsilon_{\mathrm{d} 2} \cos \omega_{\mathrm{d}} t+\frac{\lambda_{\mathrm{d} 2}}{2} \sin 2 \omega_{\mathrm{d}} t\right)$

The auxiliary vibrating beam end speed is expressed as:

$\ddot{y}_{\mathrm{d} 2}=-r_{\mathrm{d} 2} \omega_{\mathrm{d}}^{2}\left(\cos \omega_{\mathrm{d}} t-\lambda_{\mathrm{d} 2} \varepsilon_{\mathrm{d} 2} \sin \omega_{\mathrm{d}} t+\lambda_{\mathrm{d} 2} \cos 2 \omega_{\mathrm{d}} t\right)$

The main and auxiliary vibrating eccentric sleeve rotate with the eccentric shaft, and only produce rotary inertia forces, with mass $m_{\mathrm{d} 1}$ and $m_{\mathrm{d} 4}$, respectively. The main and auxiliary vibrating beam make plane motion, and produce rotary inertia force and reciprocating inertia force, with mass $m_{\mathrm{d} 2}$ and $m_{\mathrm{d} 3}$, respectively. The forces of main and auxiliary vibrating beam can decompose into the alternative forces, $m_{\mathrm{E}}$ and $m_{\mathrm{F}}$, concentrated in the main and auxiliary eccentric sleeve center, to do rotary motion, and the alternative forces $m_{\mathrm{B}}$ and $m_{\mathrm{C}}$, concentrated in the main and auxiliary vibrating end, to cause reciprocating movement.

As shown in Fig. (4), the centroid of main and auxiliary vibrating beam is not connected to the centroid and the main and auxiliary vibration connecting rod ends. The vector calculation method is used to determine the value of alternative force. The alternative centroid of the member coincides with the centroid position of the original components.

$r_{E}$ and $r_{B}$ represent the vector of the rotational center of main vibrating beam $\mathrm{E}$ and the end portion of the main vibrating link $\mathrm{D}$, respectively. Then the alternative quality of main vibrating $\mathrm{m}_{\mathrm{d} 4}$ can be expressed as:

$$
\left.\begin{array}{l}
m_{\mathrm{d} 2}=m_{\mathrm{E}}+m_{\mathrm{B}} \\
m_{\mathrm{d} 2} r_{G_{\mathrm{d} 2}}=m_{\mathrm{E}} r_{\mathrm{E}}+m_{\mathrm{B}} r_{\mathrm{B}}
\end{array}\right\}
$$

According to the trigonometric relationships in Fig. (4), the geometric equations can be obtained as follows:

$r_{G_{\mathrm{d} 2}}=r_{\mathrm{E}}+L_{E G_{\mathrm{d} 2}}$
$L_{E G_{\mathrm{d} 2}}=\left(p_{1}+i q_{1}\right) \frac{L_{\mathrm{EB}}}{l_{\mathrm{EB}}}$

where $\mathrm{L}_{\mathrm{EB}}$ can be expressed as:

$L_{\mathrm{EB}}=r_{\mathrm{E}}+r_{\mathrm{B}}$

Then, $r_{G_{\mathrm{d} 2}}$ can be expressed as:

$r_{G_{\mathrm{d} 2}}=\left(l_{\mathrm{EB}}+p_{1}+i q_{1}\right) \frac{r_{\mathrm{E}}}{l_{\mathrm{EB}}}+\left(p_{1}+i q_{1}\right) \frac{r_{\mathrm{B}}}{l_{\mathrm{EB}}}$

Eq. 12 is substituted into Eq.11

$m_{\mathrm{d} 2}\left(l_{\mathrm{EB}}+p_{1}+i q_{1}\right) \frac{r_{\mathrm{E}}}{l_{\mathrm{EB}}}+m_{\mathrm{d} 2}\left(p_{1}+i q_{1}\right) \frac{r_{\mathrm{B}}}{l_{\mathrm{EB}}}$

$=m_{\mathrm{E}} r_{\mathrm{E}}+m_{\mathrm{B}} r_{\mathrm{B}}$

Then, the alternative quality of main vibrating is shown in Eq.14.

$$
\begin{aligned}
& m_{E}=\left(\frac{l_{E B}+p_{1}}{l_{E B}}+\frac{q_{1}}{l_{E B}} i\right) m_{d 2} \\
& m_{B}=\left(\frac{p_{1}}{l_{E B}}+\frac{q_{1}}{l_{E B}} i\right) m_{d 2}
\end{aligned}
$$

Replace into the rotation center of mass of main vibration beam and eccentric sleeve and can be viewed as the rotating mass of main vibrating mechanism, which is shown in Eq.15.

$$
\begin{aligned}
m_{\mathrm{S} 1} r_{\mathrm{E}} & =m_{\mathrm{E}} r_{\mathrm{E}}+m_{\mathrm{d} 1} r_{\mathrm{E}} \\
& =\left(m_{\mathrm{d} 1}+\frac{l_{\mathrm{EB}}+p_{1}}{l_{\mathrm{EB}}} m_{\mathrm{d} 2}+\frac{q_{1}}{l_{\mathrm{EB}}} i m_{\mathrm{d} 2}\right) r_{\mathrm{E}}
\end{aligned}
$$

The rotating mass of main vibration mechanism produces centrifugal force in the outward direction along the crank, which is expressed as:

$F_{\mathrm{S} 1}=m_{\mathrm{S} 1} r_{\mathrm{E}} \omega_{\mathrm{d}}^{2}$

Replace into the end quality of main vibration beam will produce reciprocating inertial force which moves along the vertical direction, as formulated in Eq.17.

$$
\begin{aligned}
F_{\mathrm{B}}= & -m_{\mathrm{B}}\left[-r_{\mathrm{d} 1} \omega_{\mathrm{d}}^{2}\left(\cos \omega_{\mathrm{d}} t-\lambda_{\mathrm{d} 1} \varepsilon_{\mathrm{d} 1} \sin \omega_{\mathrm{d}} t\right.\right. \\
& \left.\left.+\lambda_{\mathrm{d} 1} \cos 2 \omega_{\mathrm{d}} t\right)\right]
\end{aligned}
$$

The same as the main vibrating mechanism, the alternative quality of the auxiliary vibrating mechanism can be expressed as Eq.18.

$$
\begin{aligned}
& m_{\mathrm{F}}=\left(\frac{l_{\mathrm{FC}}+p_{2}}{l_{\mathrm{FC}}}+\frac{q_{2}}{l_{\mathrm{FC}}} i\right) m_{\mathrm{d} 3} \\
& m_{\mathrm{C}}=\left(\frac{p_{2}}{l_{\mathrm{FC}}}+\frac{q_{2}}{l_{\mathrm{FC}}} i\right) m_{\mathrm{d} 3}
\end{aligned}
$$

Replace into the rotation center quality of auxiliary vibration beam and eccentric sleeve which can be viewed as 
the rotating mass of auxiliary vibrating mechanism, as formulated in in Eq.19.

$$
\begin{aligned}
m_{\mathrm{S} 2} r_{\mathrm{F}} & =m_{\mathrm{F}} r_{\mathrm{F}}+m_{\mathrm{d} 4} r_{\mathrm{F}} \\
& =\left(m_{\mathrm{d} 4}+\frac{l_{\mathrm{FC}}+p_{1}}{l_{\mathrm{FC}}} m_{\mathrm{d} 3}+\frac{q_{1}}{l_{\mathrm{FC}}} i m_{\mathrm{d} 3}\right) r_{\mathrm{F}}
\end{aligned}
$$

The rotating mass of auxiliary vibration mechanism produces centrifugal force in the outward direction along the crank, which is expressed as:

$$
F_{\mathrm{S} 2}=m_{\mathrm{S} 2} r_{\mathrm{F}} \omega_{\mathrm{d}}^{2}
$$

Replace into the end quality of auxiliary vibration beam will produce reciprocating inertia force which moves along the vertical direction, as formulated in Eq.21.

$$
\begin{aligned}
F_{\mathrm{C}}=- & m_{\mathrm{C}}\left[-r_{\mathrm{d} 2} \omega_{\mathrm{d}}^{2}\left(\cos \omega_{\mathrm{d}} t-\lambda_{\mathrm{d} 2} \varepsilon_{\mathrm{d} 2} \sin \omega_{\mathrm{d}} t\right.\right. \\
& \left.\left.+\lambda_{\mathrm{d} 2} \cos 2 \omega_{\mathrm{d}} t\right)\right]
\end{aligned}
$$

Because the main and auxiliary vibrating beams are arranged at 180 degrees to each other, and the structure arrangement is basically fixed, so it can be a reasonable assumption, based on the location and mass parameters of the main and auxiliary vibrating beam, to make the resultant forces of the rotating inertial forces and reciprocating inertial forces reduced and to reduce the influence of double vibration mechanism on the ironing plate vibration [9]. The purpose of balance of mechanism is to make the centrifugal force, generated by rotation of the main and auxiliary vibrating beam be equal and opposite in direction and cancelling the effects of each other out as formulated by eq. 22 .

$$
\left.\begin{array}{l}
F_{\mathrm{S} 1}=-F_{\mathrm{S} 2} \\
F_{\mathrm{B}}=-F_{\mathrm{C}}
\end{array}\right\}
$$

According to the above requirements, taking the amplitude of the main vibrating beam as $5 \mathrm{~mm}$, the amplitude value of the auxiliary vibrating beam is $6 \mathrm{~mm}$. Substituted into double vibrator mechanism parameters, the structure and quality parameters of main and auxiliary vibrating beams are obtained by calculation which are tabulated in Table 2. Among these, $\lambda_{\mathrm{d} 1}$ is very small, so it is ignored.

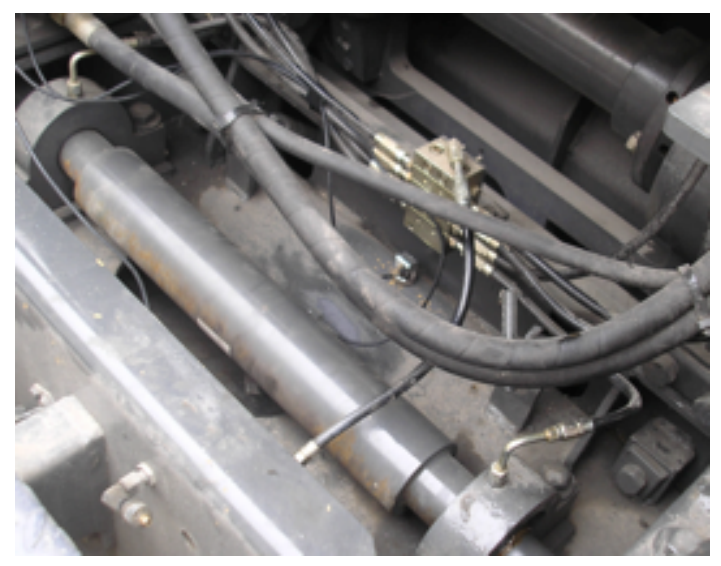

Fig. (6). Vibration test for screed.
Table 2. Phase between the main and auxiliary tamper.

\begin{tabular}{|c|c|c|c|c|}
\hline \multirow{2}{*}{ Main Vibrating Beam } & $r_{\mathrm{d} 1} / \mathrm{mm}$ & 2.5 & $m_{\mathrm{d} 2} / \mathrm{kg}$ & 90 \\
\cline { 2 - 5 } & $p_{1} / \mathrm{mm}$ & 233.19 & $q_{1} / \mathrm{mm}$ & 2.7 \\
\hline \multirow{2}{*}{ Auxiliary Vibrating Beam } & $r_{\mathrm{d} 2} / \mathrm{mm}$ & 3 & $m_{\mathrm{d} 3} / \mathrm{kg}$ & 63.21 \\
\cline { 2 - 5 } & $P_{2} / \mathrm{mm}$ & 229.84 & $q_{2} / \mathrm{mm}$ & 13.03 \\
\hline
\end{tabular}

\section{EXPERIMENT VERIFICATION}

To verify the calculation results, the real test is carried out on the screed. During the experiment, the screed frame is supported by the tires, vibration beam vacant, acceleration sensor selectively arranged on each measuring point of the framework.

According to the above optimization results, quality parameters for each section of the double-tamper mechanism of the screed are adjusted, so we do the analysis of the system at $10 \mathrm{~Hz}$ and $20 \mathrm{~Hz}$ vibration frequencies. Then, the results before and after adjustment are shown in Figs. $(\mathbf{7}, \mathbf{8})$. The L4 $\sim$ R4 represents each screed for segment arranged from left to right Fig. (6).

From the comparison of the results of double-tamper mechanism before and after parameters adjustment, we can conclude that under different vibration frequencies, the effective value of vibration acceleration of section screed centroid decreases perceptibly after screed dual tamping mechanism were improved, which is at $10 \mathrm{~Hz}$. The mean effective value of the acceleration is reduced by $43.48 \%$, at $20 \mathrm{~Hz}$, and the mean effective value of the acceleration is reduced by $43.70 \%$. Under different frequencies, the vibration standard deviation of the ironing plate frame, after improvement, was reduced, so the whole vibration uniformity is improved.

\section{CONCLUSION}

In this paper, through the analysis of the structure of the double tamping mechanism, the relationship between the amplitude and the phase angle of main and auxiliary vibrating beam is obtained. The double-tamper mechanism with two parallel slider-cranks mechanism is considered for analysis, and the theoretical calculation model of mechanism is established. Then, the inertia force balance analysis is

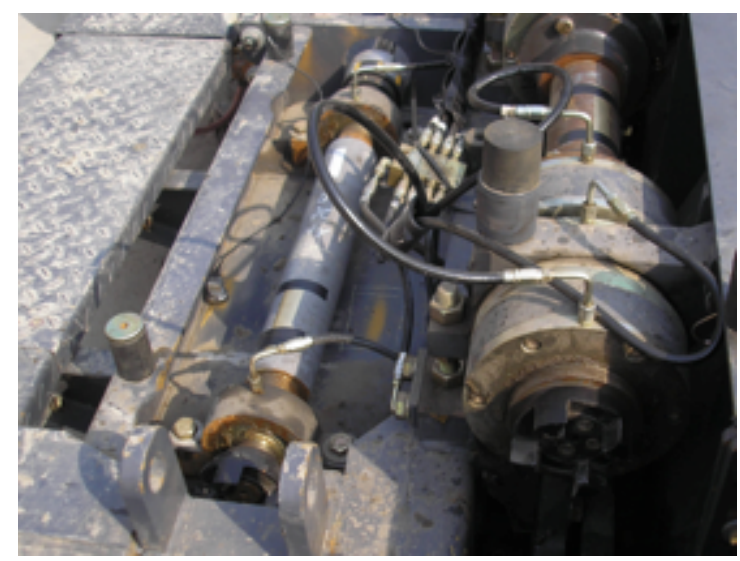



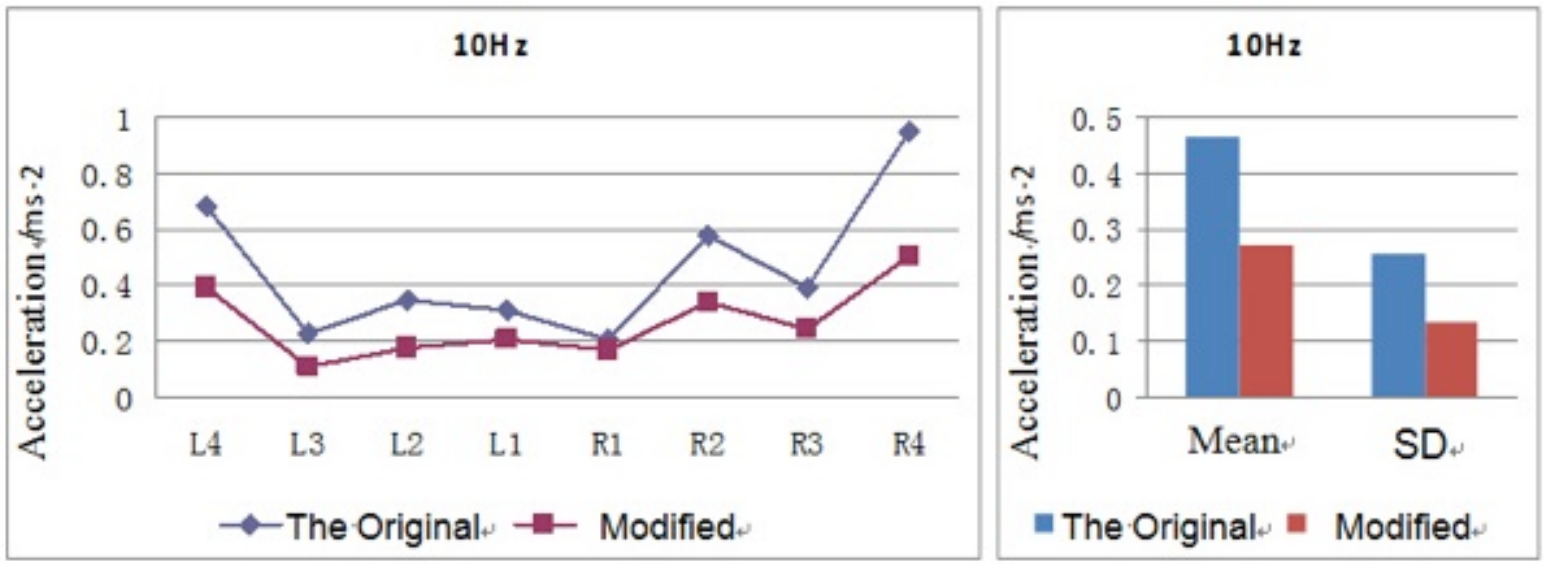

Fig. (7). $10 \mathrm{~Hz}$, inertia force comparison of mechanism.
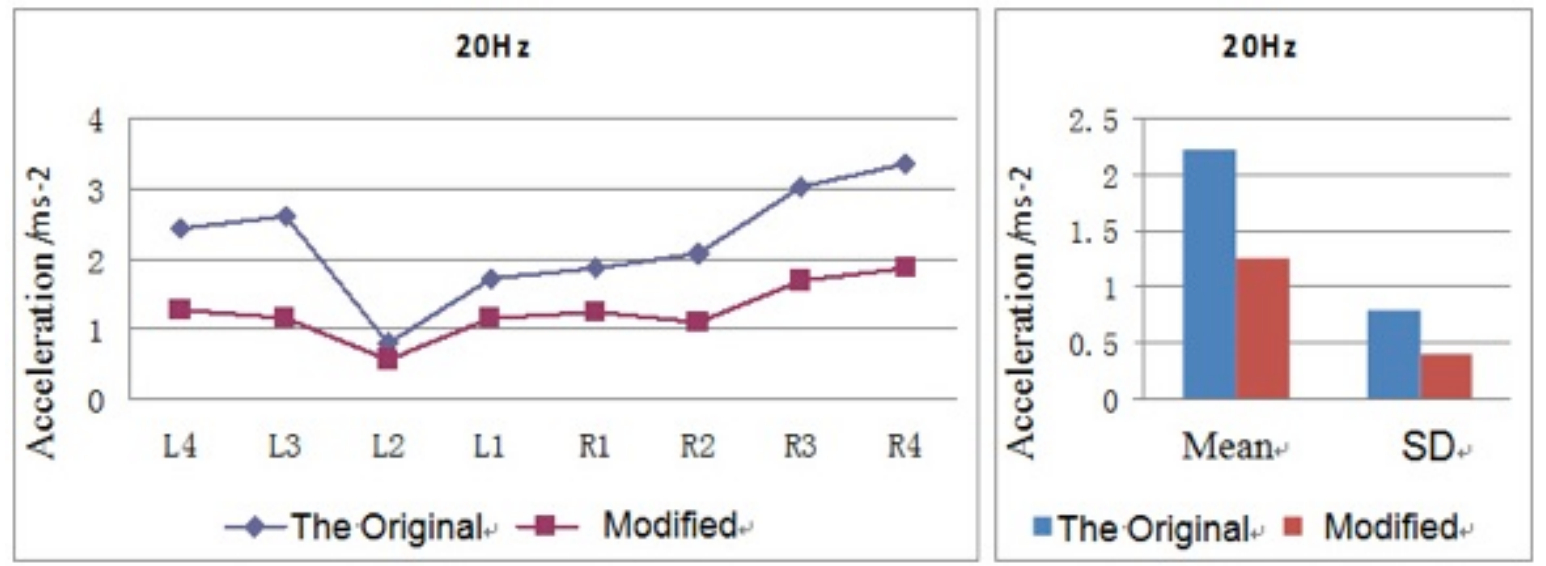

Fig. (8). $20 \mathrm{~Hz}$, Iinertia force comparison of mechanism.

carried out on the double tamper mechanism, and the structure parameters are optimized, which makes the acceleration RMS of the screed reduce by about $43 \%$, and effectively reduce the influence of vibrating mechanism on iron tablets. The research of this paper could provide a reference for the design of double-tamper mechanism for asphalt paver and the adjustment of construction parameters.

\section{CONFLICT OF INTEREST}

The author confirms that this article content has no conflict of interest.

\section{ACKNOWLEDGEMENTS}

Declared none.

\section{REFERENCES}

L. Gang, T. Jinyue, X. Zhongyu, and X. Liangquan, "Simulation of dynamic character of compacting mechanism of paver", J. Agricult. Mach., vol. 36, no. 11, pp. 34-37, 2005.
[2] Q. Xu, "Kinematic analysis of tamper mechanism for paver", Road Mach. Construct. Mechanizat., vol. 23, no. 3, pp. $15-19,2006$.

[3] G. Zhao, "Analysis on dynamic characteristic of ironing mechanism of asphalt concrete paver", Road Mach. Construct. Mechanizat., vol. 6, pp. 35-38, 2009.

[4] Z. ji, "Explanation for alphabetic marking principle on the eccentric sleeve in dual tamping mechanism of the screed", Construct. Mach. Equip., vol. 38, no. 8, pp. 11 -13, 2007.

[5] F. Zhong-xu, Z. Lin-bo, W. Xian-xue, "Dynamic simulation and parametric selection for tamping mechanism of paving machines", Chinese J. Construct. Mach., vol.7, no.1, pp.26-30, 2009.

[6] Z. Xinrong, Z. Guopu, S. Zuwang, "Dynamics analysis of screeding unit for asphalt paver", J. Xi' an Highway Univ., vol.1, pp. 95-98, 2001.

[7] Z. Shiwei, G. Zhonghua, Z. Jingliang, "Mathematical model and method of dynamic balance for two kinds of screw rotors", Int. $J$ Adv. Comput. Technol., vol. 16, no. 4, pp. 229-238. 2012.

[8] W. Jun, Z. Sheng-dun, S. Hu-shan, "A method to calculate and counterbalance the inertia force of slider-crank mechanisms in high-speed presses", Academic J. Xi'an Jiaotong Univ., vol.21, no.3, pp.141-148. 2009

[9] Q. Li-Li, C. Xiao-hui, "Balance analysis of reciprocating inertia force based on multi-body system simulation", Adv. Mat. Res., vol. 538-541, pp. 709-712, 2012 